\title{
Optimum quantum dot size for highly efficient fluorescence bioimaging
}

Cite as: J. Appl. Phys. 111, 023513 (2012); https://doi.org/10.1063/1.3676251

Submitted: 15 September 2011 . Accepted: 12 December 2011 . Published Online: 25 January 2012

Laura Martínez Maestro, Carlos Jacinto, Uéslen Rocha, M. Carmen Iglesias-de la Cruz, Francisco SanzRodriguez, Angeles Juarranz, José García Solé, and Daniel Jaque

\section{ARTICLES YOU MAY BE INTERESTED IN}

Fluorescence quantum yield of CdSe/ZnS nanocrystals investigated by correlated atomicforce and single-particle fluorescence microscopy

Applied Physics Letters 80, 4033 (2002); https://doi.org/10.1063/1.1482785

Spectrally resolved size-dependent third-order nonlinear optical properties of colloidal CdSe quantum dots

Applied Physics Letters 100, 041102 (2012); https://doi.org/10.1063/1.3679381

Size dependence of two-photon absorption in semiconductor quantum dots Journal of Applied Physics 114, 014301 (2013); https://doi.org/10.1063/1.4811349

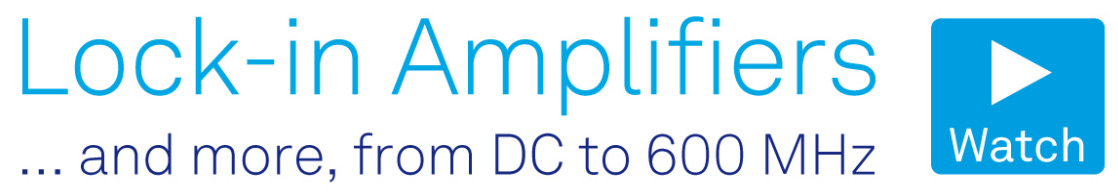




\title{
Optimum quantum dot size for highly efficient fluorescence bioimaging
}

\author{
Laura Martínez Maestro, ${ }^{1}$ Carlos Jacinto, ${ }^{2}$ Uéslen Rocha, ${ }^{2}$ M. Carmen Iglesias-de la Cruz, ${ }^{3}$ \\ Francisco Sanz-Rodriguez, ${ }^{4}$ Angeles Juarranz,${ }^{4}$ José García Solé, ${ }^{1}$ and Daniel Jaque ${ }^{1, a)}$ \\ ${ }^{1}$ Fluorescence Imaging Group, Departamento de Física de Materiales, Facultad de Ciencias, Instituto Nicolás \\ Cabrera, Universidad Autónoma de Madrid, Madrid 28049, Spain \\ ${ }^{2}$ Grupo de Fotônica e Fluidos Complexos, Instituto de Física, Universidade Federal de Alagoas, Maceió, \\ Alagoas 57072-970, Brazil \\ ${ }^{3}$ Departamento de Fisiología, Facultad de Medicina, Universidad Autónoma de Madrid, C/Arzobispo Morcillo \\ $s / n$, Madrid 29029, Spain \\ ${ }^{4}$ Departamento de Biología, Facultad de Ciencias, Universidad Autónoma de Madrid, Madrid 28049, Spain
}

(Received 15 September 2011; accepted 12 December 2011; published online 25 January 2012)

\begin{abstract}
Semiconductor quantum dots of few nanometers have demonstrated a great potential for bioimaging. The size determines the emitted color, but it is also expected to play an important role in the image brightness. In this work, the size dependence of the fluorescence quantum yield of the highly thermal sensitive CdTe quantum dots has been systematically investigated by thermal lens spectroscopy. It has been found that an optimum quantum yield is reached for 3.8-nm quantum dots. The presence of this optimum size has been corroborated in both one-photon excited fluorescence experiments and two-photon fluorescence microscopy of dot-incubated cancer cells. Combination of quantum yield and fluorescence decay time measurements supports that the existence of this optimum size emerges from the interplay between the frequency-dependent radiative emission rate and the size-dependent coupling strength between bulk excitons and surface trapping states. (C) 2012 American Institute of Physics. [doi:10.1063/1.3676251]
\end{abstract}

\section{INTRODUCTION}

Semiconductor nano-crystals, usually referred to as quantum dots (QDs), are nowadays regarded as one of the building blocks in modern photonics. $^{1-4}$ They constitute bright and photostable fluorescence sources, whose emission and absorption properties can be adequately tailored through their size. ${ }^{5}$ Recent advances on the controlled modification of their surface have made possible the development of water soluble QDs without causing deterioration in their fluorescence properties. ${ }^{6}$ This has made them excellent optical selective markers to be used in fluorescence bio-imaging experiments. ${ }^{7}$ The suitability of colloidal QDs for bioimaging is pushed forward by their large two-photon absorption cross section, ${ }^{3}$ which allows for an efficient fluorescence under infrared excitation (two-photon excitation). This, in turn, allows for large penetration depths in tissues, minimization of auto-fluorescence, and achievement of superior spatial imaging resolution. ${ }^{8}$ In addition, recent works have demonstrated the ability of QDs to act as nano-thermometers based on the thermal sensitivity of their fluorescence bands. ${ }^{9-14}$ Based on all these outstanding properties, QDs have been successfully used to mark specific receptors in cell membranes, to measure intracellular temperature, and to label living embryos at different stages. ${ }^{9,15-17}$

Most of the QD-based bio-images reported up to now were obtained by using either CdSe or CdTe QDs, since both are currently commercially available with a high degree of

\footnotetext{
a) Author to whom correspondence should be addressed. Electronic mail: daniel.jaque@uam.es.
}

quality. ${ }^{7,18-20}$ Although they show similar fluorescence properties and optical performance, CdTe QDs have very recently attracted much attention, since a hyper-thermal sensitivity of their fluorescence bands was discovered. Based on this, it has been recently postulated that intracellular thermal sensing with resolutions as high as $0.25^{\circ} \mathrm{C}$ can be achieved based on CdTe QDs, three times better than those achievable when using CdSe QDs. ${ }^{21}$

For any bio-imaging application, the fluorescence quantum yield (hereafter QY) of QDs is a key parameter that determines the fluorescence brightness in real applications. QY is defined as the ratio between the number of emitted and absorbed photons per unit time. This is determined by the radiative and non-radiative decay rates $\left(k_{\text {rad }}\right.$ and $k_{\text {nrad }}$, respectively) in such a way that QY $=k_{\text {rad }} /\left(k_{\text {rad }}+k_{\text {nrad }}\right)$. The QY does not only determine the fluorescence brightness of the QDs (and, hence, the bio-image contrast), but also, and even more important, it determines the pump-induced thermal loading of the biological system, which is under local illumination. QDs with low QY would have large nonradiative de-excitation rates and, hence, could cause relevant thermal loadings that can alter the normal evolution of the biomedical system during imaging. As a consequence, the search of those parameters maximizing the fluorescence QY of QDs is an open question with undoubted practical applications and fundamental implications. Among the different parameters that could be used for QY tailoring, size is very likely the most critical one. Previous works concerning fluorescence decay time dynamics of QDs have provided evidence that both $k_{\text {rad }}$ and $k_{\text {nrad }}$ are expected to be strongly size-dependent. ${ }^{22-28}$ On one side, it is known that $k_{\text {rad }}$ 
decreases with the QD size. This fact arises from the relation between the radiative rate, $k_{r a d}$, and the emission frequency peak (determined by QD size) established by the Fermi's "golden rule" of pure exciton states, but also affected by the participation of the so-called "dark exciton states". ${ }^{22,27}$ On the other side, it is now widely assumed that the nonradiative decay rate, $k_{\text {nrad }}$, in QDs is related to the presence of surface acceptor energy states. ${ }^{27,29}$ Thus, this nonradiative decay rate would be determined by the density of acceptor states (expected to increase with the surface-to- volume ratio, i.e., as the QD size is reduced) and also by the coupling strength between these acceptor states and the intrinsic electronic levels of the QDs. This last factor is favored as the size of QDs is increased, because a weaker quantum confinement makes energy separation between electronic states smaller (facilitating coupling and migration). Therefore, both $k_{\text {rad }}$ and $k_{\text {nrad }}$ (and, hence, QY) are predicted to be strongly dependent on the QD size, so that an adequate choice of it could lead to the optimum QY. Thus, a systematic study on the size effect in the fluorescence QY of CdTe QDs is necessary to determine if the predictable optimum size occurs. This could be of particular interest for biomedical imaging in order to choose the most indicated fluorescent QDs probes.

In this work, we have systematically investigated, by thermal lens spectroscopy (TLS), the fluorescence QY of colloidal CdTe QDs with sizes ranging from 1 up to $8 \mathrm{~nm}$. The existence of an optimum size, leading to a maximum in the QY, has been corroborated by one-photon excited fluorescence experiments as well as by two-photon fluorescence imaging of epithelial cancer cells (HeLa cells). In addition, the combination of the TLS results with fluorescence lifetime measurements has allowed us to determine how both $k_{\text {rad }}$ and $k_{\text {nrad }}$ of CdTe QDs contribute to the size dependence of the fluorescence QY.

\section{EXPERIMENTAL}

The CdTe QDs used in this work were provided by Plasmachem Inc. and were all obtained in identical condi-

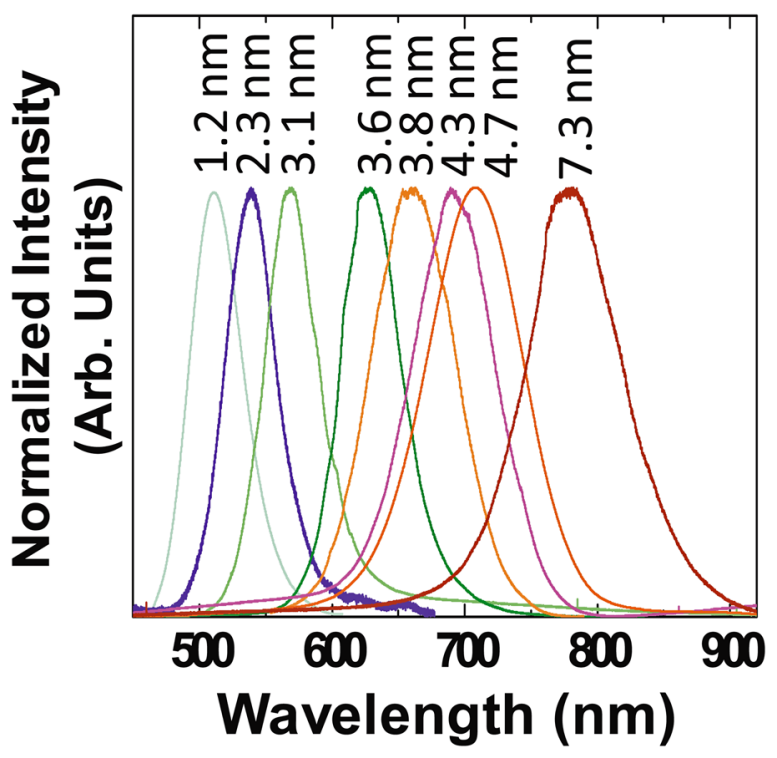

FIG. 1. (Color online) Normalized emission spectra of different QDs used in this work. Excitation wavelength was $488 \mathrm{~nm}$. tions through an aqueous synthesis without phase transfer. Up to eight different CdTe QDs were investigated, with sizes ranging from 1.2 up to $8 \mathrm{~nm}$. The nominal dot sizes provided by the manufacturer were obtained from the analysis of the spectroscopic properties, following a previous work of $\mathrm{Yu}$ et al. ${ }^{30} \mathrm{CdTe}$ QDs were dispersed in distilled water with a concentration of $0.3 \%$ by mass without any evidence of precipitation/deterioration during months.

The emission spectra of the solutions were measured in a fluorescence confocal microscope under identical geometrical conditions. The excitation was carried out at $488 \mathrm{~nm}$, provided by an argon laser (Spectra-Physics 177-G02), and the emission spectra was recorded using a CCD coupled to a monochromator.

For fluorescence lifetime measurements, we used a nitrogen-pulsed laser $(337 \mathrm{~nm}, 5 \mathrm{~ns})$ as excitation source. The decay time profile of the subsequent luminescence was recorded by a Hamamatsu R5108 photomultiplier detector connected to a digital oscilloscope.

HeLa cells were incubated for two hours in a biological phosphate buffer solution (PBS), containing a fixed concentration ( $0.3 \%$ in weight) of CdTe QDs. The images included
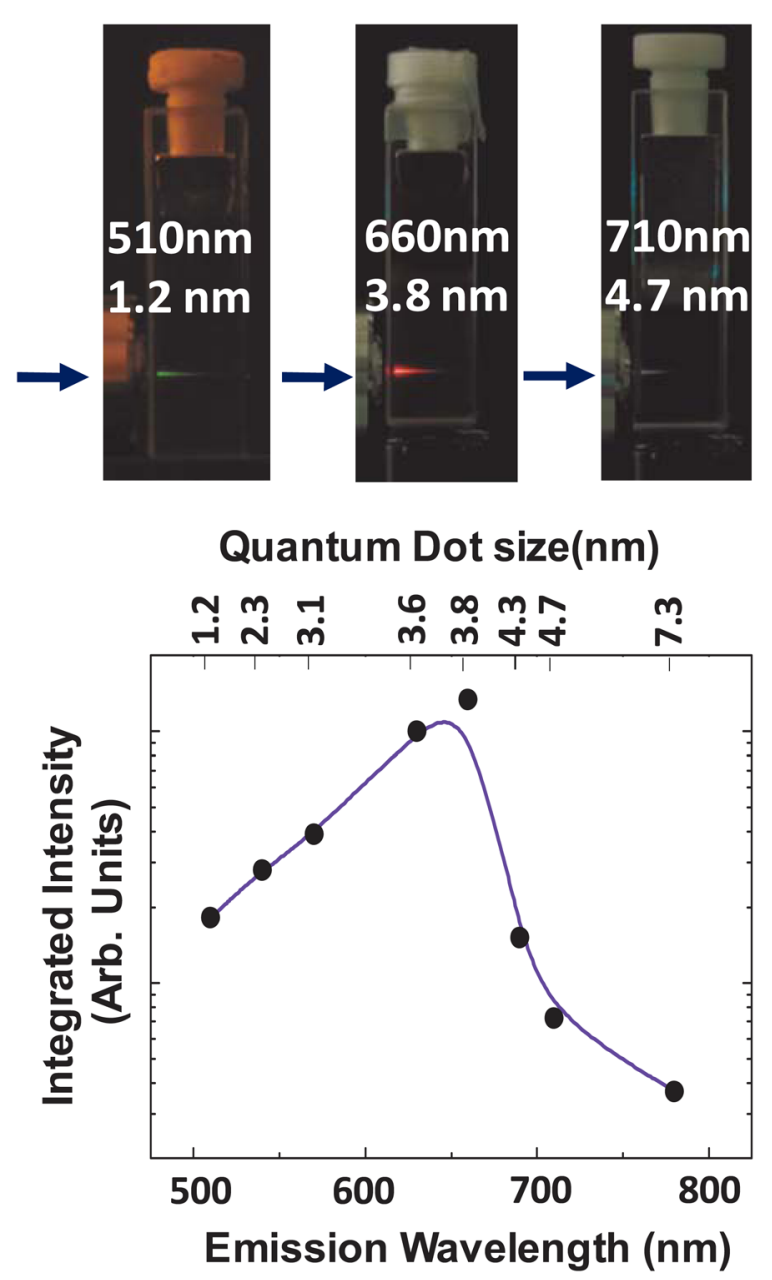

FIG. 2. (Color online) Top: Digital pictures of three representative CdTe QDs solutions. Both the peak wavelength and QD size are indicated in each case. Arrows represent the 488-nm excitation beam. Bottom: Emitted intensity as a function of the peak wavelength and QD size. Dots are experimental data and solid line is an eye guide. 

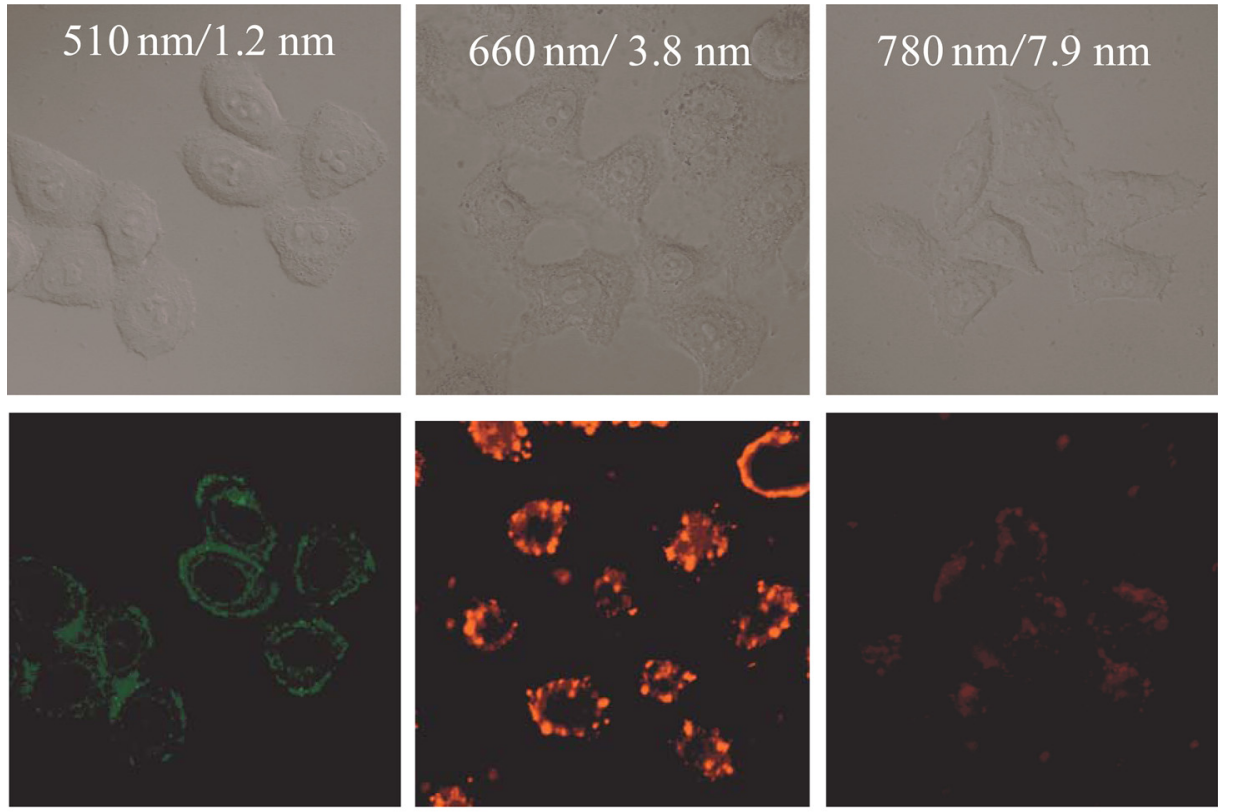

FIG. 3. (Color online) Two-photon fluorescence images of HeLa cancer cells as obtained by using CdTe QDs with three different representative sizes. The optical transmission images of the HeLa cells are at the top, whereas the two-photon excited fluorescence and the superimposed images constitute the middle and bottom rows, respectively.
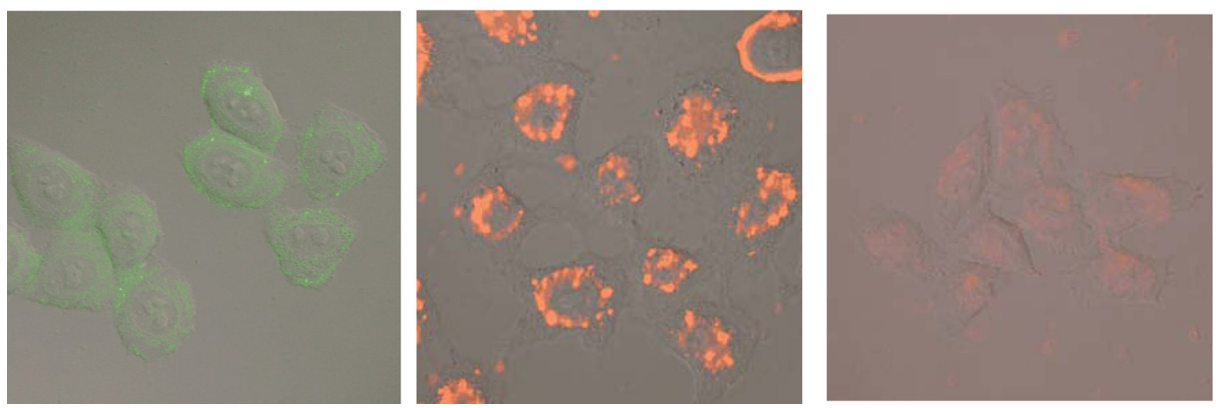

in Fig. 3 were obtained with the same excitation intensity at $800 \mathrm{~nm}$ by a Mai Tai femtosecond pulsed laser (SpectraPhysics).

For the thermal lens measurements, an argon laser was used for the pump beam and a He-Ne $(632 \mathrm{~nm})$ laser for the probe beam. The signal was recorded using an oscilloscope.

\section{RESULTS AND DISCUSSION}

All of the CdTe QDs solutions showed an intense fluorescence under continuous-wave one-photon excitation at $488 \mathrm{~nm}$. Figure 1 shows the normalized emission spectra of the CdTe QDs solutions used in this work. Each solution produces a different emission peak wavelength according to the confinement, due to its particular dot size (also indicated). It should be noted that not all the solutions showed the same fluorescence brightness. This is clearly evidenced in Fig. 2 (top), which shows digital pictures of three representative CdTe QDs solutions obtained under optical excitation at $488 \mathrm{~nm}$. It can be appreciated that CdTe QDs with a diameter of $3.8 \mathrm{~nm}$ showed a most intense brightness than those of $4.7 \mathrm{~nm}$ and $1.2 \mathrm{~nm}$. In order to quantify this, the emission spectra of the solutions (all of them with the same weight \% content) were measured in a fluorescence confocal microscope under identical geometrical conditions. The obtained spectra were corrected by the spectral response of the detection system and normalized by the absorbed pump power at the excitation volume. Results are shown in Fig. 2 at the bottom. In agreement to what is observed by the naked eye, $660 \mathrm{~nm}$ (3.8-nm dot size) CdTe QDs showed the best pumpto-fluorescence conversion efficiency. The existence of this "optimum size" has been also found in two-photon excited fluorescence bioimaging experiments. Figure 3 shows images of HeLa cancer cells taken by two-photon excited fluorescence microscopy obtained after $2 \mathrm{~h}$ of incubation in a biological phosphate buffer solution (PBS) containing a fixed concentration ( $0.3 \%$ in weight) of CdTe QDs. The three selected samples correspond to relevant QDs' sizes: $3.8 \mathrm{~nm}$ (optimum size); $1.2 \mathrm{~nm}$ (the smallest one); and $8 \mathrm{~nm}$ (the biggest one). All the images included in Fig. 3 have been obtained with the same $800 \mathrm{~nm}$ excitation intensity and after applying to all of them the same image treatments (contrast and brightness). Thus, it is clear that the cell images with highest contrast were obtained when the $3.8 \mathrm{~nm}$ CdTe QDs were used. This fact indicates that the optimum fluorescence efficiency has been reached for this particular size (in accordance with what was observed from the analysis of the one-photon excited emission). As it will be shown next, the existence of this "optimum size" is, indeed, supported by the size-variation of the QY obtained from TLS experiments.

As described elsewhere, TLS is based on a simple pump-and-probe set-up, which is schematically shown in Fig. 4. ${ }^{31-33}$ Basically, the QDs solution is locally heated by a time-modulated beam (the pump beam) that is partially absorbed by the CdTe QDs. In our case, this "pump beam" was provided by a slightly focused argon laser, and the heat 

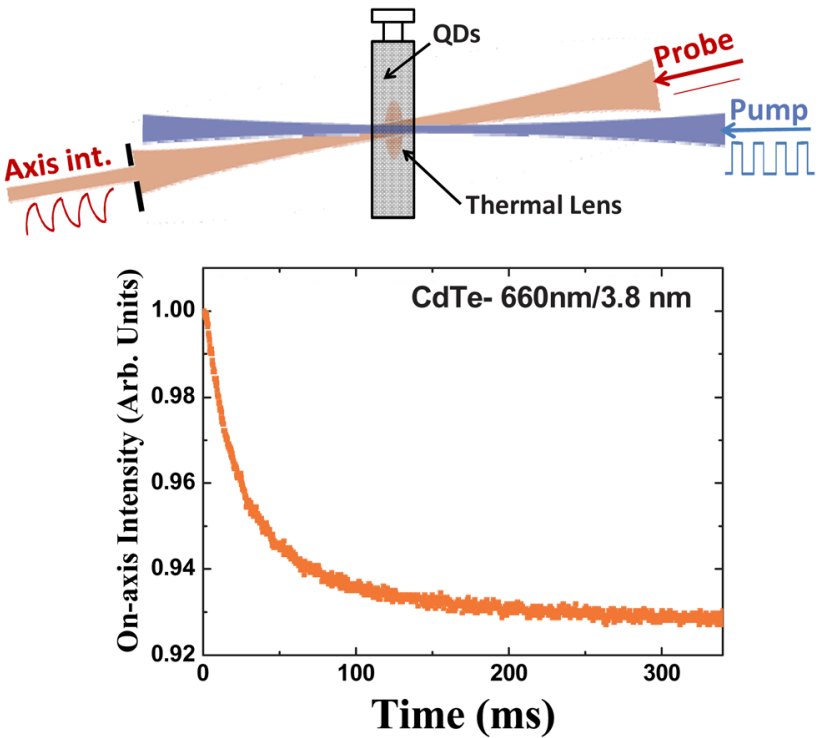

FIG. 4. (Color online) Top Schematic drawing of the experimental set-up used for TLS measurements. Bottom Typical on-axis transient obtained for the probe beam in our experimental set-up.

delivered is due to the non-vanishing $k_{\text {nrad }}$. This local heating leads to the appearance of a time-modulated thermal lens (TL). The power of this TL (which is determined by the fraction of absorbed photons that de-excite via non-radiative processes) is determined by measuring the distortion induced in a low-power, non-modulated "beam" (the so-called "probe beam") that is almost collinear with the pump one. In our case, this probe beam was delivered by a He-Ne $632 \mathrm{~nm}$ laser. The TL-induced focusing power of the probe beam is then monitored by measuring its on-axis intensity that, due to the modulated nature of the pump beam, displays a "transient" behavior. Figure 4 shows a typical on-axis decay curve obtained in our experimental set-up for the $660 \mathrm{~nm}-3.8 \mathrm{~nm}$ CdTe QDs. From the analysis of the relation between the amplitude of the transient signal and the excitation power and wavelength, it is possible to determine the fluorescence QY without the requirement of any reference sample, as detailed elsewhere. ${ }^{34}$ This technique was applied to all the QDs solutions, and the obtained sizedependence of the fluorescence QY is shown in Fig. 5. In this figure, two different regimes are clearly observed. For QDs' diameters larger than $3.8 \mathrm{~nm}$ (emission wavelengths larger than $660 \mathrm{~nm}$ ), the fluorescence QY has been found to decrease as the QD size (emission peak) is increased. On the other hand, for QDs smaller than $3.8 \mathrm{~nm}$ (i.e., emission wavelengths shorter than $660 \mathrm{~nm}$ ), the QY has been found to increase monotonously with size increasing (i.e., increasing peak emission wavelength). This behavior was also observed by M. Grabolle et al., who measured the QY of different CdTe QDs in the size range 2-3.5 $\mathrm{nm}$ by using reference dye samples. ${ }^{24}$ We now clearly see that the crossover between the two observed regimes (see Fig. 5) occurs for a QD size of $3.8 \mathrm{~nm}$. For this "critical" size, the QY scales up to 0.47 , in reasonable agreement with previous works that report on QY values ranging from 0.25 up to 0.6 for CdTe QDs. ${ }^{11,23,35}$ At this point, it should be noted that a small variation in

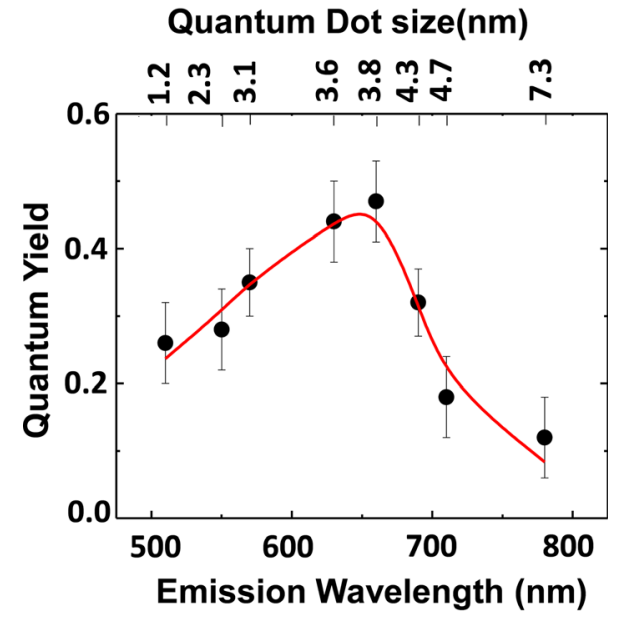

FIG. 5. (Color online) Fluorescence quantum yield of CdTe QDs solutions as a function of QD emission wavelength (dot size). Dots are experimental data and solid line is an eye guide.

synthesis conditions may result in a large difference in quantum efficiency, even with the same experimental conditions, but different batches. Nevertheless, we ruled out this possibility by repeating the measurements, using completely different batches provided by the same company. The same results of Fig. 5 (within the uncertainty there indicated) were obtained. The maximum QY value found for the $3.8 \mathrm{~nm}$ QDs unequivocally indicates that, for this particular size, the ratio of emitted to absorbed photons reaches a maximum. This is, indeed, in qualitative agreement with both the normalized fluorescence measurements and the bioimaging experiments that also settle on the optimum QD size at $3.8 \mathrm{~nm}$ (see Figs. 2 and 3 ).

In order to get a better understanding on the sizedependence of QY, we performed fluorescence lifetime measurements. The fluorescence decay curves did not fluctuate with the collection time, i.e., successive measurements lead to the same decay time curve shape. This result indicates that "blinking effects" are not detected in our experiments. ${ }^{36}$ The fluorescence decay curves of the smallest QDs (with time constants close to $10 \mathrm{~ns}$ ) were not measured in our experimental set-up, because they are close to our time resolution.

At this point, it is important to mention that the "decay time dynamics" of QDs are not still completely understood. Because of the so-called "blinking effect", the decay-time curve shape of a single quantum dot fluctuates with the collection time. Sophisticated time-tagged, time-resolved measurements performed on single CdSe quantum dots have shown that these fluctuations are due to fluctuations in the non-radiative rate rather than in the radiative rate. ${ }^{36}$ As a consequence, when the time decay curve of an assembly of QDs displays a non-single exponential decay curve, the long-time component is associated to the radiative decay, whereas the fast component is caused by the presence of non-radiative processes. Therefore, in this work, we have estimated the radiative decay possibility as the inverse of the long-time "tail" component of our decay curves. ${ }^{25,36}$ According to the long-term component, the radiative rates estimated 


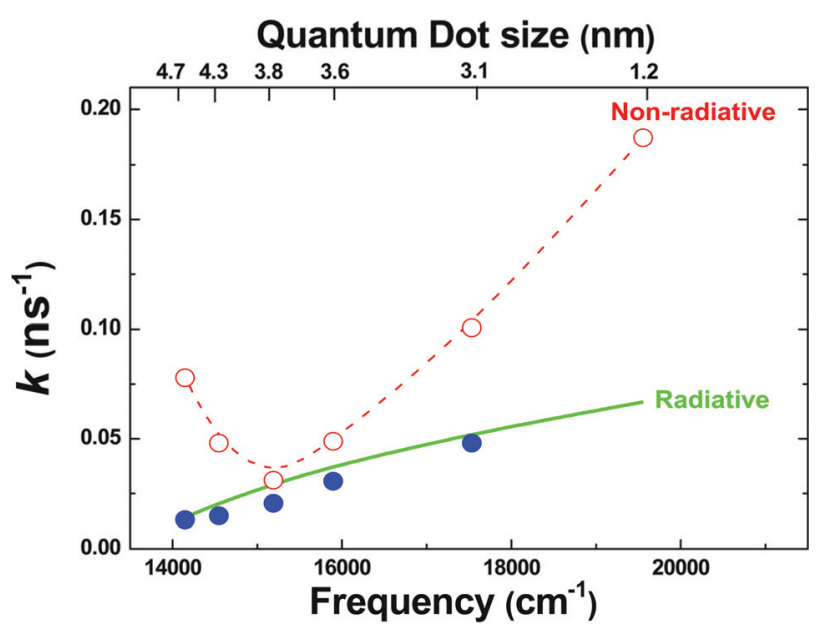

FIG. 6. (Color) Radiative and non-radiative decay rates of all the CdTe QDs solutions under study in this work and plotted as a function of size and central emission frequency. Solid line is the radiative decay rate calculated according with Ref. 27. Solid dots are the radiative decay rates calculated from the exponential tail of the decay curves. The non-radiative decay rates obtained by combining the radiative decay rates and the quantum yield values included in Fig. 5 are indicated by the open dots. Dashed line is an eye guide.

in such a way (i.e., from the fluorescence decay curves) are in fairly good agreement with the radiative rates previously calculated and measured for CdTe nanocrystals. ${ }^{27}$ This is evidenced in Fig. 6, where the radiative rates experimentally determined from our fluorescence lifetime measurements are displayed together with those predicted by van Driel et al. ${ }^{27}$ These radiative decay rates together with the QY values independently measured by TLS (Fig. 5) were used to determine the non-radiative decay rates of all the CdTe QD solutions based on the relation $k_{\text {nrad }}=\left(\mathrm{QY}^{-1}-1\right) \cdot k_{\text {rad }}$. The obtained $k_{\text {nrad }}$ values are also included in Fig. 6. A careful inspection of this figure reveals that, whereas $k_{\text {rad }}$ increases monotonously with the emitted frequency (decreasing size), $k_{\text {nrad }}$ shows two well-defined regimes. For emission frequencies below $15000 \mathrm{~cm}^{-1}$ (dots larger than $3.8 \mathrm{~nm}$ ), $k_{\text {nrad }}$ decreases with increasing frequency (decreasing dot size). On the contrary, for frequencies above that of $3.8 \mathrm{~nm}$ dots, this non-radiative rate strongly increases with emission frequency (i.e., with decreasing dot size). Results included in Fig. 6 can satisfactorily explain the two-regime size-dependence of QY observed in Fig. 5. We state that the regime corresponding to a "decreasing of QY with dot size" (for dots larger than $3.8 \mathrm{~nm}$ ) is mostly caused by an enhancement in the coupling strength between surface acceptor states and intrinsic electronic states that leads to larger non-radiative decay probabilities. This is reasonable, since larger dots correspond to a weaker quantum confinement, so that energy separation between intrinsic electronic CdTe states is reduced. Consequently, migration to surface states becomes more probable. On the other hand, the regime corresponding to a "decrease in the QY while reducing QD size" (from 3.8 down to $1.2 \mathrm{~nm}$, emission frequencies above $15000 \mathrm{~cm}^{-1}$ ) can be attributed to a dramatic increase in the non- radiative rate. This enhancement in the non-radiative rate is due to an increase in the relative density of surface trap states that should occur as a consequence, increasing the surface-to-volume ratio.

\section{CONCLUSIONS}

In summary, we have clearly demonstrated the existence of an optimal QD size that optimizes the fluorescence QY of CdTe QDs. Thermal lens spectroscopy measurements have concluded that CdTe QDs $3.8 \mathrm{~nm}$ in diameter show the highest fluorescence QY (close to 0.5 ), so that they would provide the highest contrast with minimum thermal loading of the bio-specimen under imaging. The existence of this "optimum size" has been corroborated in both simple onephoton excited fluorescence experiments and in two-photon excited fluorescence microscopy experiments with cancer cells incubated with CdTe quantum dots. The optimum size has been explained in terms of size-induced changes in both the radiative and non-radiative decay rates.

The results here presented open a new avenue in the search of new fluorescence "multifunctional nanoprobes" for high-resolution fluorescence imaging. Recall that these nanoparticles are not only highly efficient, but they also present the dual function of two-photon excited fluorescence imaging and, at the same time, fluorescence nanothermometry. Thus, the use of these optimum nanoprobes will provide the possibility of detecting new static and dynamic biomedical phenomena at the nanoscale.

\section{ACKNOWLEDGMENTS}

This work was supported by the Universidad Autónoma de Madrid and Comunidad Autonoma de Madrid (Projects CCG087-UAM/MAT-4434 and 2009/MAT-1756), by the Spanish Ministerio de Educacion y Ciencia (MAT 201016161), by a Banco Santander CEAL-UAM project, and by the Brazilian agencies CAPES, CNPq, and FAPEAL (through the PRONEX 2009-09-006).

${ }^{1}$ A. P. Alivisatos, J. Phys. Chem. 100, 13226 (1996).

${ }^{2}$ V. L. Colvin, M. C. Schlamp, and A. P. Alivisatos, Nature 370, 354 (1994).

${ }^{3}$ V. I. Klimov, A. A. Mikhailovsky, S. Xu, A. Malko, J. A. Hollingsworth, C. A. Leatherdale, H. J. Eisler, and M. G. Bawendi, Science 290, 314 (2000).

${ }^{4}$ P. V. Kamat, J. Phys. Chem. C 112, 18737 (2008).

${ }^{5}$ L. Brus, J. Phys. Chem. 90, 2555 (1986).

${ }^{6}$ D. R. Larson, W. R. Zipfel, R. M. Williams, S. W. Clark, M. P. Bruchez, F. W. Wise, and W. W. Webb, Science 300, 1434 (2003).

${ }^{7}$ X. Michalet, F. F. Pinaud, L. A. Bentolila, J. M. Tsay, S. Doose, J. J. Li, G. Sundaresan, A. M. Wu, S. S. Gambhir, and S. Weiss, Science 307, 538 (2005). ${ }^{8}$ C. Xu, W. Zipfel, J. B. Shear, R. M. Williams, and W. W. Webb, Proc. Natl. Acad. Sci. U.S.A. 93, 10763 (1996).

${ }^{9}$ L. M. Maestro, E. M. Rodriguez, F. S. Rodriguez, M. C. I. la Cruz, A. Juarranz, R. Naccache, F. Vetrone, D. Jaque, J. A. Capobianco, and J. G. Sole, Nano Lett. 10, 5109 (2010).

${ }^{10}$ P. A. S. Jorge, M. Mayeh, R. Benrashid, P. Caldas, J. L. Santos, and F. Farahi, Meas.Sci. Technol. 17, 1032 (2006).

${ }^{11}$ G. W. Walker, V. C. Sundar, C. M. Rudzinski, A. W. Wun, M. G. Bawendi, and D. G. Nocera, Appl. Phys. Lett. 83, 3555 (2003).

${ }^{12} \mathrm{~S}$. Li, K. Zhang, J. M. Yang, L. W. Lin, and H. Yang, Nano Lett. 7, 3102 (2007).

${ }^{13}$ J. C. Kim, H. Rho, L. M. Smith, H. E. Jackson, S. Lee, M. Dobrowolska, and J. K. Furdyna, Appl. Phys. Lett. 75, 214 (1999).

${ }^{14}$ B. Han, W. L. Hanson, K. Bensalah, A. Tuncel, J. M. Stern, and J. A. Cadeddu, Ann. Biomed. Eng. 37, 1230 (2009). 
${ }^{15}$ J. R. Slotkin, L. Chakrabarti, H. N. Dai, R. S. E. Carney, T. Hirata, B. S. Bregman, G. I. Gallicano, J. G. Corbin, and T. F. Haydar, Dev. Dyn. 236, 3393 (2007).

${ }^{16}$ J. Lovric, H. S. Bazzi, Y. Cuie, G. R. A. Fortin, F. M. Winnik, and D. Maysinger, J. Mol. Med. 83, 377 (2005).

${ }^{17}$ K. M. Fichter, M. Flajolet, P. Greengard, and T. Q. Vu, Proc. Natl. Acad. Sci. U.S.A. 107, 18658 (2010).

${ }^{18}$ W. C. W. Chan, D. J. Maxwell, X. Gao, R. E. Bailey, M. Han, and S. Nie, Curr. Opin. Biotechnol. 13, 40 (2002).

${ }^{19}$ H. Arya, Z. Kaul, R. Wadhwa, K. Taira, T. Hirano, and S. C. Kaul, Biochem. Biophys. Res. Commun. 329, 1173 (2005).

${ }^{20}$ W. Jiang, E. Papa, H. Fischer, S. Mardyani, and W. C. W. Chan, Trends Biotechnol. 22, 607 (2004)

${ }^{21}$ L. M. Maestro, C. Jacinto, U. R. Silva, F. Vetrone, J. A. Capobianco, D. Jaque, and J. G. Sole, Small 7, 1774 (2011).

${ }^{22}$ G. Schlegel, J. Bohnenberger, I. Potapova, and A. Mews, Phys. Rev. Lett. 88, 137401 (2002).

${ }^{23}$ S. F. Wuister, I. Swart, F. van Driel, S. G. Hickey, and C. D. Donega, Nano Lett. 3, 503 (2003).

${ }^{24}$ M. Grabolle, M. Spieles, V. Lesnyak, N. Gaponik, A. Eychmuller, and U. Resch-Genger, Anal. Chem. 81, 6285 (2009).
${ }^{25}$ Y. Ebenstein, T. Mokari, and U. Banin, Appl. Phys. Lett. 80, 4033 (2002).

${ }^{26}$ N. O. Dantas, P. M. N. de Paula, R. S. Silva, V. Lopez-Richard, and G. E. Marques, J. Appl. Phys. 109, 024308 (2011).

${ }^{27}$ A. F. van Driel, G. Allan, C. Delerue, P. Lodahl, W. L. Vos, and D. Vanmaekelbergh, Phys. Rev. Lett. 95, 236804 (2005).

${ }^{28}$ M. D. Leistikow, J. Johansen, A. J. Kettelarij, P. Lodahl, and W. L. Vos, Phys. Rev. B 79, 045301 (2009).

${ }^{29}$ V. Fomenko and D. J. Nesbitt, Nano Lett. 8, 287 (2008).

${ }^{30}$ W. W. Yu, L. Qu, W. Guo, and X. Peng, Chem. Mater. 15, 2854 (2003).

${ }^{31}$ C. Jacinto, D. N. Messias, A. A. Andrade, S. M. Lima, M. L. Baesso, and T. Catunda, J. Non-Cryst. Solids 352, 3582 (2006).

${ }^{32}$ R. A. Cruz, V. Pilla, and T. Catunda, J. Appl. Phys. 107, 083504 (2010).

${ }^{33}$ J. Shen, R. D. Lowe, and R. D. Snook, Chem. Phys. 165, 385 (1992).

${ }^{34}$ M. L. Baesso, J. Shen, and R. D. Snook, Chem. Phys. Lett. 197, 255 (1992).

${ }^{35}$ S. V. Kershaw, M. Burt, M. Harrison, A. Rogach, H. Weller, and A. Eychmuller, Appl. Phys. Lett. 75, 1694 (1999).

${ }^{36}$ B. R. Fisher, H. J. Eisler, N. E. Stott, and M. G. Bawendi, J. Phys. Chem. B 108, 143 (2004) 